\title{
A terahertz molecular switch
}

\author{
P. A. Orellana ${ }^{1}$ and F. Claro ${ }^{2}$ \\ ${ }^{1}$ Departamento de Física, Universidad Católica del Norte, Casilla 1280, Antofagasta, Chile \\ ${ }^{2}$ Facultad de Física, Pontificia Universidad Católica de Chile, Casilla 306, Santiago 22, Chile
}

\begin{abstract}
We present time-dependent results describing the current through a molecular device, modeled as a complex with two active centers connected to leads under bias. We show that, at a properly adjusted external voltage, a passing terahertz electromagnetic pulse may cause a transition between states of finite and negligible current, suggesting that the system might be useful as a nanoscopic switch in the terahertz range. A phase diagram defining the bias region in which the transition takes place within a short time is given. As described, the physical processes involved are of an entirely different nature than those in ordinary photodetectors.
\end{abstract}

PACS numbers: 73.40.Gk, 73.63.-b, 85.65.+h, 74.78.Na

Individual molecules as well as molecular complexes are increasingly being perceived as possible electronic device elements, constituting great promise in the process of miniaturization. Some successes have already been reported along this line $e^{1.2 .3 .4}$. Among recent examples are molecules that exhibit big on-off current ratios and a large negative differential resistance in two-terminal transport ${ }^{5.6}$, behaving much in the way some mesoscopic semiconductor tunneling heterostructures do ${ }^{7.8}$. Such performance may be understood decomposing the system into coupled molecular subunits that transit collectively through a conducting quantum resonance as the bias along the device is modified.10.11. The currentvoltage profile is then determined by the properties of this resonance, which in turn may depend strongly on the dynamic accumulation of electronic charge in the various subunits, thus affecting the tunneling rate for transport.

When a pair of neighboring subunits of a different kind are involved, an energy level associated with one site must reach alignment with one in the other site in order for a device resonance to be established. The narrow voltage range in which significant current flows in the experimental data of Chen et $a^{15}$ suggests that such mechanism may be at work in some molecules. Using this two site model, Han et al found qualitative agreement between the model predictions and the experimental data ${ }^{9}$. A time dependent approach for the analysis of the same model allowed us to fit the data to an impressive degree of accuracy, and showed in addition that switching between distinct current states is possible, as reported below. Switching is extremely fast compared to that in organic complexes, where the effect has been assigned to local structural disorder induced by the external electric field ${ }^{12}$. While in the latter switching times are of the order of $0.1 \mu \mathrm{s}$, we here report characteristic times in the order of $0.1 \mathrm{ps}$, six orders of magnitude shorter. The device is then sensitive to terahertz pulses, suggesting that the system is potentially useful as a nanoscopic detector of electromagnetic radiation in the $\mathrm{THz}$ range.

We consider a molecular complex connected to leads subject to an external dc bias potential. As shown schematically in Fig. 1, the complex is modeled by two active sites, connected to left and right particle reservoirs
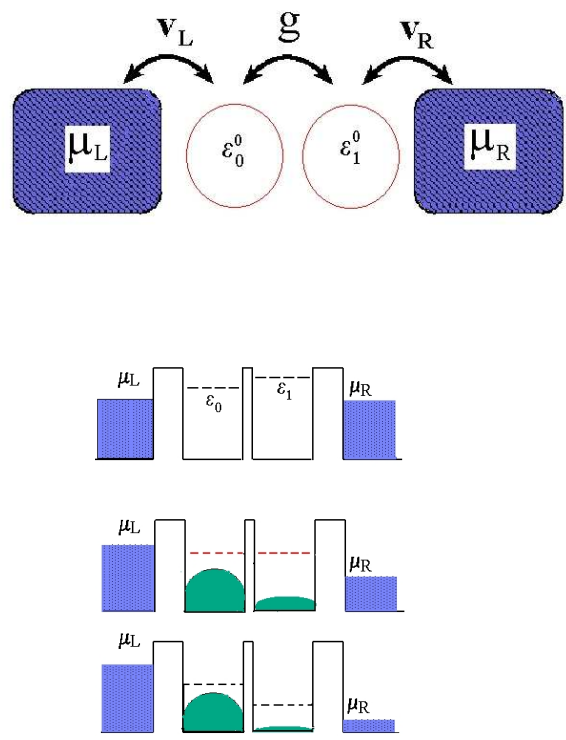

FIG. 1: Scheme of the device. a, the molecule is placed between leads at chemical potentials $\mu_{L}$ and $\mu_{R}$. It is characterized by two active sites with energies $\varepsilon_{0}^{o}, \varepsilon_{1}^{o}$, which under bias, b, change their relative positions allowing for the resonance condition to be established in a certain bias range. The shaded areas show the amount of electron charge at each site.

at chemical potentials $\mu_{L}$ and $\mu_{R}$, respectively. The device is described by the Hamiltonian, 


$$
\begin{aligned}
H= & \sum_{i \neq 0,1 ; \sigma} \varepsilon_{i} n_{i \sigma}+t \sum_{i(\neq 0,1) ; \sigma}\left(c_{i, \sigma}^{\dagger} c_{i+1, \sigma}+c_{i+1, \sigma} c_{i, \sigma}^{\dagger}\right)+\sum_{\alpha=0,1 ; \sigma} \varepsilon_{\alpha} n_{\alpha, \sigma}+v_{L} \sum_{\sigma}\left(c_{-1, \sigma}^{\dagger} c_{0, \sigma}+c_{0, \sigma}^{\dagger} c_{-1, \sigma}\right) \\
& +v_{R} \sum_{\sigma}\left(c_{1, \sigma}^{\dagger} c_{2, \sigma}+c_{2, \sigma}^{\dagger} c_{1, \sigma}\right)+g \sum_{\sigma}\left(c_{0, \sigma}^{\dagger} c_{1, \sigma}+c_{1, \sigma}^{\dagger} c_{0, \sigma}\right)+U \sum_{\alpha=0,1} n_{\alpha_{\uparrow}} n_{\alpha_{\downarrow}}
\end{aligned}
$$

where $c_{i \sigma}^{\dagger}\left(c_{i \sigma}\right)$ creates (destroys) an electron with spin $\sigma$ at site $\mathrm{i}$ and $n_{i, \sigma}=c_{i \sigma}^{\dagger} c_{i \sigma}$ represents the occupation of site $i$. The molecular sites are labeled 0 and 1 and have intrinsic energies $\varepsilon_{0}^{o}$ and $\varepsilon_{1}^{o}$, respectively, and an intra-molecular coupling $g$. The coupling strength of the molecule to the left (right) lead is $v_{L(R)}$, while t represents the hoping parameter within the leads. The total site energy $\varepsilon_{i}$ includes the fixed intrinsic energy at the site, the external radiation-induced voltage and the applied dc bias, the latter represented by a classical term linear in the spatial coordinate $i$. Finally, the effect of charge build-up at the molecular sites is accounted for by a non-linear term with coupling constant $\mathrm{U}$, the last one in the above expression, that leaves out occupation with the same quantum numbers. The time-dependent wave function that evolves according to this Hamiltonian can be expanded in a tight-binding basis as

$$
\left|\psi_{k \sigma}(\tau)\right\rangle=\sum_{i} a_{i \sigma}^{k}(\tau)\left|\phi_{i \sigma}\right\rangle
$$

where $\left|\phi_{i \sigma}\right\rangle$ is a Wannier state of spin $\sigma$ localized at site $i$, and the coefficients $a_{i \sigma}^{k}(\tau)$ obey the non-linear equations

$$
\begin{aligned}
i \hbar \frac{d a_{j, \sigma}^{k}}{d \tau} & =\varepsilon_{j}(\tau) a_{j, \sigma}^{k}+t\left(a_{j-1, \sigma}^{k}+a_{j+1, \sigma}^{k}\right) \quad(j \neq-1,0,1,2), \\
i \hbar \frac{d a_{-1(2), \sigma}^{k}}{d \tau} & =\varepsilon_{-1(2)}(\tau) a_{-1(2), \sigma}^{k}+v_{L(R)} a_{0(1), \sigma}^{k}+t a_{-2(3), \sigma}^{k}, \\
i \hbar \frac{d a_{0, \sigma}^{k}}{d \tau} & =\left(\varepsilon_{0}(\tau)+U\left\langle n_{0}\right\rangle\right) a_{0, \sigma}^{k}+v_{L} a_{-1(1), \sigma}^{k}+g a_{1, \sigma}^{k} \\
i \hbar \frac{d a_{1, \sigma}^{k}}{d \tau} & =\left(\varepsilon_{1}(\tau)+U\left\langle n_{1}\right\rangle\right) a_{1, \sigma}^{k}+v_{R} a_{2, \sigma}^{k}+g a_{0, \sigma}^{k} .
\end{aligned}
$$

In this expression the average site densities equal $\left\langle n_{0}\right\rangle=$ $\sum_{k, \sigma}\left|a_{0, \sigma}^{k}\right|^{2},\left\langle n_{1}\right\rangle=\sum_{k, \sigma}\left|a_{1, \sigma}^{k}\right|^{2}$, where the sum over $k, \sigma$ covers all occupied electron states. In writing Eqs. (3) we have adopted a Hartree model for the electronelectron interaction. As we will show in what follows, the terms proportional to the site densities generated by this interaction, nonlinear in nature, are of key importance in the behavior of the system.

Solutions are found discretizing equations (3) and using a half-implicit numerical method, which is secondorder accurate and unitary ${ }^{14.15}$. It is assumed that the coefficients outside the structure are given at incident wavenumber $k$ by the expressions

$$
\begin{gathered}
a_{j, \sigma}^{k}=\left(I e^{i k j a}+R_{j} e^{-i k j a}\right) e^{-i \varepsilon \tau / \hbar} \quad(j a \leq-L) \\
a_{j, \sigma}^{k}=T_{j} e^{i k^{\prime} j a} e^{-i \varepsilon \tau / \hbar} \quad(j a \geq L)
\end{gathered}
$$

where the device is explicitly defined to lie between sites $-L$ and $L, a$ is the lattice constant, and $k^{\prime}=$ $\left[2 m(\varepsilon-V) / \hbar^{2}\right]^{1 / 2}$ is the wavenumber of the transmitted particle, with $V=\mu_{L}-\mu_{R}$. The incident amplitude $I$ is supposed to be spatially constant. Also, far from the molecule the amplitude of the reflected and transmitted waves $R_{j}$ and $T_{j}$ are supposed to be weakly dependent on site $j$. This permits to restrict the dependence to the linear term, which is found to be an adequate approximation provided the time step taken to discretize equations (3) is less than certain limit value that depends upon the parametrization of the system. In the numerical procedure the Wannier amplitudes obtained for one bias are used as the starting point for the next bias step. Once these coefficients are known the current is calculated from ${ }^{15}$,

$$
J_{j}=\frac{e}{h} t \sum_{\sigma} \int_{o}^{k_{f}} \operatorname{Im}\left(a_{j, \sigma}^{k *} a_{j+1, \sigma}^{k}\right) d k
$$

We apply the above formalism to a specific situation. An excellent fit to the experimental I-V curve at 


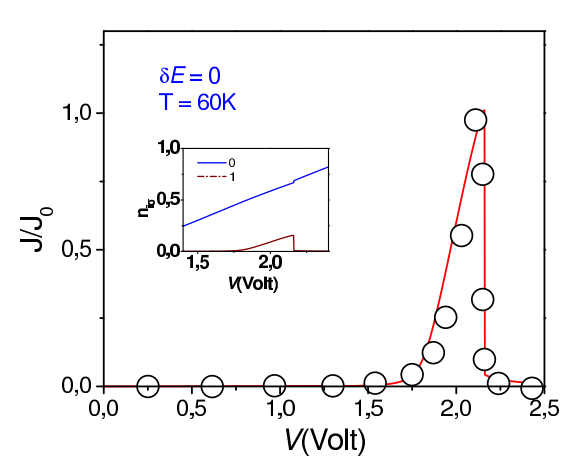

FIG. 2: Current-voltage characteristic in the absence of external radiation. Parameter values are given in the text, and were chosen to fit the experimental data of Chen et $\mathrm{a}^{{ }^{5}}$ at $\mathrm{T}=60 \mathrm{~K}$ shown as circles. The inset shows the charge in each molecule site at different bias values.

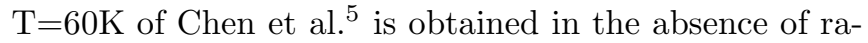
diation within this model, by choosing the parameters $\varepsilon_{0}^{o}=0.52, \varepsilon_{1}^{o}=0.92, g=0.008, t=2.0, U=0.8$ and intrinsic level broadenings $\Gamma_{L}=\Gamma_{R}=0.16$, all in units of eV. Here $\Gamma_{L(R)}=\pi v_{L(R)}^{2} \rho(0)$, with $\rho(0)$ the density of states at the Fermi energy. The energies at the active sites are represented by $\varepsilon_{i}=\varepsilon_{i}^{o}-[V+\delta E \sin (2 \pi \nu \tau)] / 2$, $(i=0,1)$, where $\delta E$ and $\nu$ are the amplitude and frequency of the incoming $\mathrm{THz}$ radiation, respectively. Energies are measured with respect to the Fermi energy at the emitter contact $\left(\mu_{L}\right)$. Figure 2 shows our results, together with experimental data points from Ref. 5 shown as circles. Notice that the current peak is quite narrow, and there is a steep fall in the upper critical voltage edge $V_{c}=2.16 \mathrm{~V}$. As the bias is increased and the first site energy approaches the Fermi energy charge begins to flow, first into site 0 , then to site 1 as well as shown in the inset and, schematically, in Fig. 1b. The effect of this charge is to raise the local potential at each subunit, thus affecting the transport coefficient of the overall quantum mechanical structure. The abrupt fall at $V_{c}$ is caused by a sudden loss of charge at site 1 , and subsequent break of the energy level alignment that allows for resonant tunneling through the molecule.

Figure 3 shows the time evolution of the current flowing through the system (Fig. 3a) and the charge density at sites 0 (full line) and 1 (broken line) (Fig. 3b) when electromagnetic radiation of frequency $\nu=10 \mathrm{THz}$ and strength $\delta E=1.8 \mathrm{mV}$ is turned on at time $=0$. The offset bias $\delta V=V_{c}-V$ has the value $0.2 \mathrm{mV}$. Before the radiation enters the sample the system is in resonance, current flows and both sites have significant charge. At $\tau=0$ the external field is turned on and after a time of the order of a picosecond, site 1 looses its charge and the current drops to a low value. This behavior illustrates the fast switching response of the device as a $\mathrm{THz}$ pulse passes by. The actual transition time is controlled by the internal coupling constant, so that in our case $T \sim \hbar / g \sim 0.08 p s$. There are small oscillations driven by the external field,

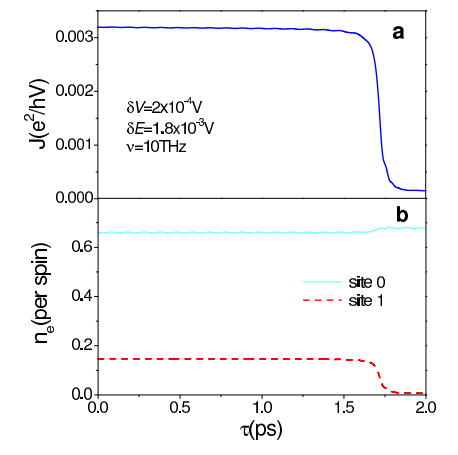

FIG. 3: Time evolution of (a) the overall current and (b) the charge density in sites 0 and 1 at $\nu=10 \mathrm{THz}$. Parameters used are as in Fig. 2.

which we have found to become larger in amplitude when the signal strength is increased. The fact that the system undergoes a few coherent oscillations before falling suggests that the random fluctuations caused by a finite temperature are not capable of overcoming the inertia of the device. Notice that in the low current state site 1 has lost its charge. This feature reveals that the system is bi-stable very close to $V_{c}$. When site 1 is charged, the local potential is dynamically adjusted to keep the system in resonance. A disturbance may cause the flow of charge out of the site, however, with the result that the resonance condition is lost and current stops.

Suppose now that the system is in a given state of high current, biased slightly below $V_{c}$. The transition to a low current state is then possible provided the amplitude of the external field is large enough to bring the system to criticality. Figure 4 shows the region in which the transition happens within 2 picoseconds time (labeled YES), separated from that region in which the radiation is too weak to cause the current to fall that fast, if at all (labeled NO). The frequency is $\nu=10 \mathrm{THz}$ and the bias is shown in terms of the offset from criticality $\delta V=V_{c}-V$. A rough fit to the boundary is given by $\delta E=0.014 \times \delta V^{1 / 2}$, all quantities in units of V. Our numerical methods allowed testing frequencies up to order $1 / \mathrm{T}$, and within that range we found the boundary to shift to higher values as the frequency increases. In the figure, the filled circle is a point in the boundary for $\nu=20 \mathrm{THz}$ while the triangle is for $\nu=5 \mathrm{THz}$. This is to be expected since in the low frequency limit one should have $\delta E \sim \delta V$.

In summary, we have shown that switching between different current states in transport through molecules is possible and may be triggered by a passing electromagnetic pulse with characteristic times in the terahertz range. Depending on the applied external bias, the passage of current is turned off by the radiation pulse, thus allowing the device to act as a tunable detector of terahertz radiation. Switching occurs because of the existence of bi-stable states near the I-V characteristic highvoltage edge. A similar bi-stable region is known to oc- 


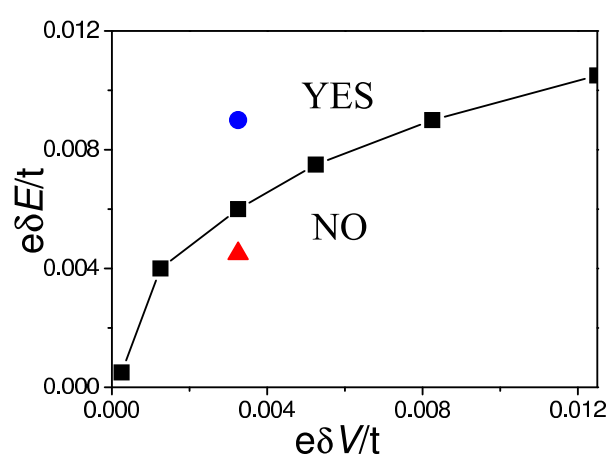

FIG. 4: Boundary separating the region at which switching takes place within 2 ps of the arrival of an external pulse, labeled YES, from the region in which the well remains charged and current still flows beyond that time interval, labeled NO. $\delta V$ measures the external bias relative to the critical edge $V_{c}$, and $\delta E$ is the radiation strength, both in units of the lead hopping parameter t. Squares are for incoming radiation of frequency $\nu=10 \mathrm{THz}$. Filled circle and triangle are for frequencies $\nu=20 \mathrm{THz}$ and $5 \mathrm{THz}$, respectively. cur in transport through GaAlAs double barrier resonant tunneling devices 15.16 .17 .18 . A single active energy level localized in the quantum well formed between the barriers is responsible for the bi-stability in such case. Near the bottom of the emitter conduction band a current carrying state through this level, lifted in energy by the charge trapped in the well, may coexist with a state of no current at the same bias with the well uncharged. In a two-site molecular device as the one treated here the effect derives from the dynamic coupling between the sites within the molecule, and thus has a different physical origin.

We thank Dr. J.E. Han for valuable discussions and bringing the work of Chen et al to our attention, and Dr. M. Orszag for comments on the manuscript. This work was supported in part by FONDECYT grants No. 1020269 and No. 1020829, Programa Milenio ICM P 99135-F(PO) and Cátedra Presidencial en Ciencias (FC).
${ }^{1}$ C. Dekker, Physics Today 52, 22 (May, 19991)

2 C. Joachim, A. Gimzewski \& Aviram, Nature 408, 541 (2000)

3 H. Park, A. Pasupathy, J. Goldsmith, C. Chang,Y. Yaish, J. Petta, M. Rinkoski, J. Sethna, H. Abruña, P. McEuen and D. C. Ralph, Nature 417, 722 (2002)

${ }^{4}$ W. Liang, M.P. Shores, M. Bockrath, J. R. Long and H. Park, Nature 417, 725 (2002)

5 J. Chen, M.A. Reed, A.M. Rawlett and J.M. Tour, Science 286, 1550 (1999)

6 J. Chen and M. A. Reed, Chem. Phys. 281, 127 (2002)

7 L. Esaki, Phys. Rev. 109, 603 (1958)

8 R. Tsu and L. Esaki, Appl. Phys. Lett. 22, 562 (1973)

9 J.E. Han and Vicent H. Crespi, Appl. Phys. Lett. 79, 2829 (2001)

10 S. Datta, W. Tian, S. Hong, R. Reifenberger, J.I. Henderson and C.P.Kubiak, Phys. Rev. Lett 79, 2530 (1997)
11 M. H. Hettler, H. Schoeller and W. Wenzel, Euro Phys. Lett. 57, 571 (2002)

12 H.J. Gao, K. Sohlberg, Z.Q. Xue, H.Y. Chen, S.M. Hou, L.P. Ma, X.W. Fang, S.J. Pang, S.J. Pennycook, Phy. Rev. Lett. 84, 1780 (2000)

13 P. Orellana, F. Claro and E. Anda, Phys. Rev. B62, 9959 (2000)

14 R. Mains and G. Haddad, J. Appl. Phys 64, 3564 (1988).

15 P. Orellana, E. Anda and F. Claro, Phys. Rev. Lett. 79 1118 (1997)

16 V. J. Goldman, D. C. Tsui and J. E. Cunningham, Phys. Rev. Lett. 58, 1256 (1987)

17 A.D. Martin, M. L.F. Lerch, P.E. Simmonds and L. Eaves, Appl. Phys. Lett. 64, 1248 (1994)

18 J. Inkoferer, G. Obermair and F. Claro, Phys. Rev. B64, 201405 (2001) 